\title{
New Emergence Mechanism Caused by Human Fuzzy Rationality
}

\author{
Ihor Lubashevsky \\ University of Aizu \\ Aizu-Wakamatsu, Fukushima, Japan \\ i-lubash@u-aizu.ac.jp
}

\author{
Arkady Zgonnikov \\ University of Aizu \\ Aizu-Wakamatsu, Fukushima, Japan \\ arkady.zgonnikov@gmail.com
}

\author{
Dmitry Parfenov \\ General Physics Institute, RAS \\ Moscow, Russia \\ narytyan@gmail.com
}

\begin{abstract}
A new emergence mechanism related to the bounded capacity of human cognition is considered. It assumes that individuals (operators) governing the dynamics of a certain system try to follow an optimal strategy in controlling its motion but fail to do this perfectly because similar strategies are indistinguishable for them, which is called human fuzzy rationality. The main attention is focused on the systems where the optimal dynamics implies the stability of a certain equilibrium point in the corresponding phase space. The human fuzzy rationality gives rise to some neighborhood of the equilibrium point, the region of dynamical traps, wherein each point is regarded as an equilibrium one by the operators. This approach is also extended to take into account the effect of imperfect implementation of the desired control strategy and a non-Newtonian model capturing this effect is developed. In particular, it is demonstrated that complex emergent phenomena can be caused by the fuzzy rationality of human behavior.
\end{abstract}

Keywords- fuzzy rationality; human cognition; dynamical trap; instability; emergence

\section{INTRODUCTION}

During the last decades there has been a great deal of modeling social systems and human behavior using physical formalism (for a review see, e.g., articles of Encyclopedia [1]). In particular, it concerns one of the basic notions in physics, the stationary point of a dynamical system. The corresponding fundamental questions about the behavior of a given system are its dynamics near the stationary point and emergent phenomena occurring via its instability. For example, the notions of a fixed-point attractor as a stable stationary point, latent attractors, periodic attractors representing limit cycles, and deterministic chaos are widely met in social psychology [2].

In spite of the gained achievements the mathematical theory of social systems seems to be currently at its initial stage of development. Indeed, animate beings and objects of the inanimate world are highly different in their basic features, in particular, such notions as willingness, learning, prediction; motives for action, moral norms are just inapplicable to inanimate objects. This enables us to pose a question as to what individual physical notions and mathematical formalism should be developed to describe social systems in addition to the available ones inherited from modern physics.

Previously the concept of dynamical traps was introduced to describe the bounded capacity of human cognition in evaluating events, actions, etc. according to their preference and its effects in governing a certain system or entity $[3,4,5]$. It was partly stimulated by studying the car following dynamics for bounded rational drivers [6,7]. When, for example, two actions are close to each other in quality from the standpoint of a human (operator) making a decision their choice may be random because he ought to consider them equivalent. The notion of dynamical traps accounts for this feature. Broadly speaking, it is an alternative to the notion of stationary point in dynamical systems [5].

Recent advances in the field of human control also have given evidence to the fact that humans do not generally operate the systems under their control in a precise way. Maintaining the system exactly at the desired position requires the ability of the operator to keep perfect awareness and to react immediately even to the smallest deviations. Meanwhile, experimental studies have revealed that the considerable response latency and the effects of noise in the sensorimotor system prevent human operators from implementing continuous control strategies (see, e.g., [8] and references therein). Instead, the discontinuous or intermittent control is found to be efficient in the presence of time delays and random perturbations in human-controlled processes [9]. The "drift and act" pattern of human control has been detected, e.g., in aircraft landing [10], stick balancing at the fingertip [11] and postural control during quiet standing [12].

\section{DYNAMICAL TRAP MODEL}

At the starting point of model development let us consider a system whose dynamics is described in the twodimensional phase space $\mathbb{R}_{x y}=\{x, y\}$ and assume that if the operator was able to govern the system perfectly following a certain optimal strategy then its dynamics would be governed by the coupled equations 


$$
\tau \frac{d x}{d t}=F_{x}(x, y), \tau \frac{d y}{d t}=F_{y}(x, y)
$$
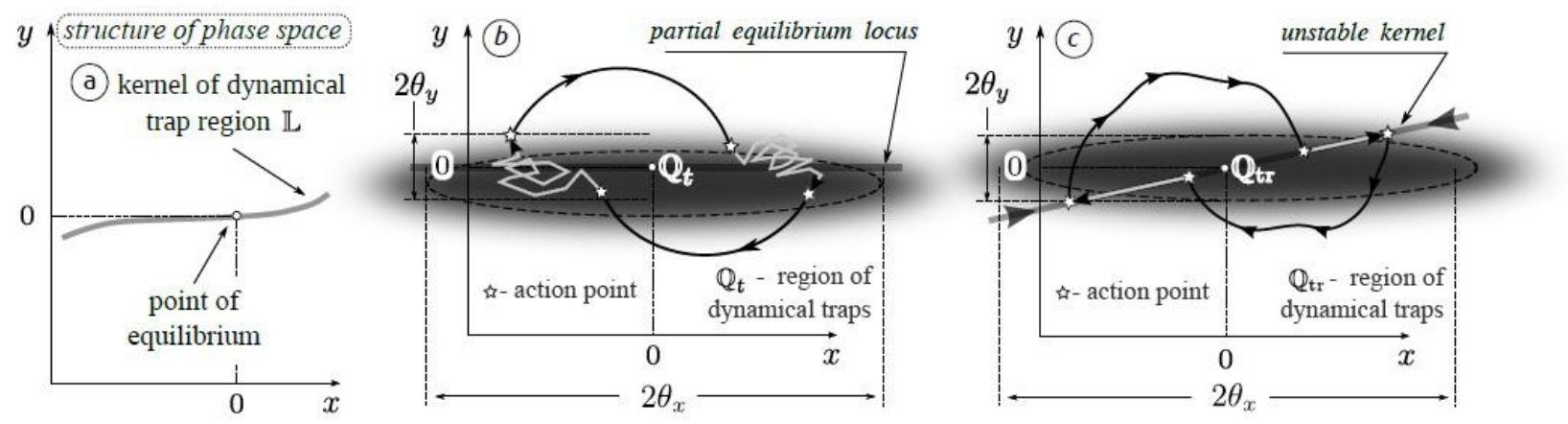

Figure 1. The structure of the phase space $\mathbb{R}_{x y}$ of the system under consideration (a) and illustration of system trajectories in the case of dynamical traps with a partial equilibrium locus (b) and a unstable kernel (c).

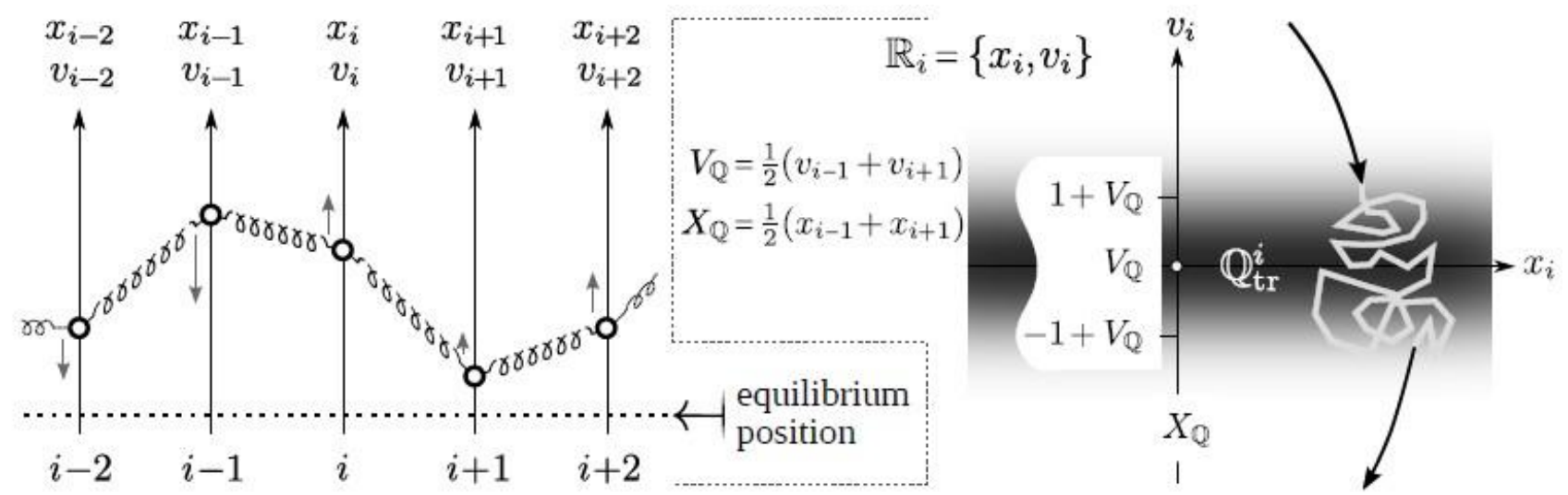

Figure 2. The chain of $\mathrm{N}$ beads and the structure of their individual phase space $\mathbb{R}_{i}=\left\{x_{i}, v_{i}\right\}(i=1,2, \ldots, N)$. The formal initial $i=0$ and terminal $i=$ $N+1$ beads are assumed to be fixed, specifying the equilibrium bead position.

Here $\tau$ is a time scale characterizing the operator perception delay, the "forces" $F_{x}(x, y)$ and $F_{y}(x, y)$ are determined by both the physical regularities of the system mechanics and the active behavior of the operator in controlling the system motion. The origin $\{0,0\}$ of the coordinate frame is placed at the equilibrium point of system (1). In this context the perfect rationality of the operator means his ability to locate precisely the current position of the system on the phase plane $\mathbb{R}_{x y}$, to predict strictly its further motion, and, then, to correct the current motion continuously. Exactly in this case it is possible to consider that the operator orders the strategies of behavior according to their preference and then chooses the optimal one. As a result the equilibrium point $\{0,0\}$ must be stable when the aim of operator actions is to keep the system in close vicinity to this point.

The motion of the given system has been presumed to be a cumulative effect of the physical regularities and the operator actions. The notion of dynamical trap kernel implements this feature. Namely, the operator is considered to be able to halt the system motion at a certain multitude $\mathbb{L}$ of points in the phase space $\mathbb{R}_{x y}$ to be called the locus of partial equilibrium $\mathbb{L}_{\text {pe if after getting any point of }} \mathbb{L}_{\text {pe the }}$ system will stay at it without furthers actions of the operator. In this case the system motion along $\mathbb{L}_{\text {pe }}$ is due to random factors or the operator action. If after the operator suspending his control over the system its dynamics becomes unstable with a relatively small increment, the multitude $\mathbb{L}$ is called the unstable kernel $\mathbb{L}_{\mathrm{uk}}$. The two cases differ from each other only in the mechanism forcing the system to leave a certain neighborhood of $\mathbb{L}$ called the dynamical trap region $\mathbb{Q}_{t r}$; due to the bounded capacity of operator cognition he cannot distinguish between its points and the point of $\mathbb{L}$. In the former case it is random or uncontrollable motion of the system (Fig. 1b); in the latter case it is the weak system instability without the operator control (Fig. 1c). The given coordinate frame is chosen so that the partial equilibrium

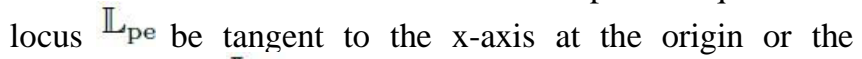
unstable kernel $\mathbb{L}_{\mathrm{uk}}$ correspond to small variations in $y$.

The system motion outside $\mathbb{Q}_{\text {tr }}$ is governed by the active behavior of the operator and the physical regularities. In the given work the continuous approximation of action points is 
used [5] mimicking the transition from active to passive behavior of the operator by the factor

$$
\Omega(x, y)=\frac{\Delta+\left(x / \theta_{x}\right)^{2}+\left(y / \theta_{y}\right)^{2}}{1+\left(x / \theta_{x}\right)^{2}+\left(y / \theta_{y}\right)^{2}}
$$

such that in the region $\mathbb{Q}_{\text {tr }}$ it takes small value $\Delta \ll 1$ whereas outside $\mathbb{Q}_{\operatorname{tr}}$ it is about unity. The thresholds $\theta_{x}$ and $\theta_{y}$ determine the dimensions of the region $\mathbb{Q}_{\mathrm{tr}}$, namely, $\mathbb{Q}_{\mathrm{tr}}=|x| \lesssim \theta_{x}$ and $|y| \lesssim \theta_{y}$

Considering, for example, systems with partial equilibrium locus the following model called a chain of oscillators with dynamical traps

$$
\frac{d v_{i}}{d t}=-\Omega\left(\vartheta_{i}\right)\left[\eta_{i}+\sigma \vartheta_{i}+\sigma_{0} v_{i}\right]+\epsilon \xi_{i}(t)
$$

can be derived under rather general assumptions [5]. Here $v_{i}=d x_{i} / d t$ is the velocity of particle (bead), the variable $\eta_{i}=x_{i}-\frac{1}{2}\left(x_{i-1}+x_{i+1}\right)$ is the position of particle $i$ with respect to its nearest neighbors, and $\vartheta_{i}=v_{i}-\frac{1}{2}\left(v_{i-1}+v_{i+1}\right)_{\text {is its relative velocity. }}$ The kinetic coefficients $\sigma$ and $\sigma_{0}$ quantify the relative weights of the stimuli for particle $i$ to get the middle position between the nearest neighbors and to keep up the relative velocity equal to zero. The last term in (3) allows for uncontrollable factors in the system dynamics treated as random noise $\xi_{\mathrm{i}}(\mathrm{t})$ with the amplitude $\epsilon$. The cofactor

$$
\Omega(\vartheta)=\frac{\Delta+\vartheta^{2}}{1+\vartheta^{2}}
$$

Describes the dynamical trap effect causing the system stagnation in $\mathbb{Q}_{\text {tr }}$ treated as a layer $|\vartheta| \lesssim 1$ in the phase plane $\{\eta, v\}$. Roughly speaking when the relative velocity $v$ of a given particle gets low values it prefers to do nothing because the situation cannot become worse too fast. The system stagnation in $\mathbb{Q}_{\text {tr }}$ is due to the small value of $\Omega(0)=\Delta \ll 1$

Using model (3) it has been demonstrated that dynamical traps can cause this system to undergo non-equilibrium phase transitions of a new type where noise plays a constructive role whereas the regular "force" does not exhibit a change in its structure similar to the bifurcation of stationary points [3]. The virtual experiments on balancing over-damped inverted pendulum also provide evidence for the model of oscillators with dynamical traps [13].

\section{Results of Numerical Simulation}

Model (3) was studied numerically. The system dynamics was found to depend on the intensity of "dissipation" quantified by the parameter $\sigma$. We remind that the parameter $\sigma$ specifies the relative weight of the stimuli to take the middle "optimal" position and to eliminate the relative velocity; the larger the parameter $\sigma$, the more significant the latter stimulus. Without noise the system tends to get the regime of regular dynamics represented by a collection of limit cycles of individual bead motion when the parameter $\sigma$ is not too small. However, the ltime to formation" $T_{N}$, i.e. the mean time required for a given bead chain to get the steady state regular dynamics grows exponentially as the number of beads $N$ increases. For example, for beads with $\sigma=1$ this time can be approximated by the function

$$
T_{N} \approx T_{c} \cdot \exp \left\{N / N_{c}\right\} \quad \text { with } T_{c} \sim 60 \text { and } N_{c} \sim 13
$$
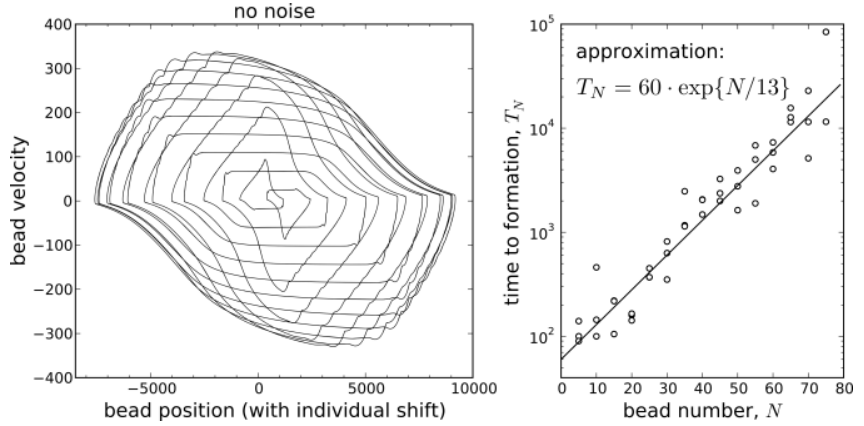

Figure 3. The characteristic phase portrait of the steady state dynamics exhibited by systems without noise and not too weak "dissipation" (left frame). The chain of 30 beads with $\sigma=1$ was used in constructing the shown pattern where the limit cycles of each second bead are visualized. The right frame depicts the characteristic time $T_{N}$ required for such a system to get the steady state dynamics vs the number $N$ of beads. The scatted points are the data obtained for each value of $N$ on three trials, $\sigma=1$ was used in simulation
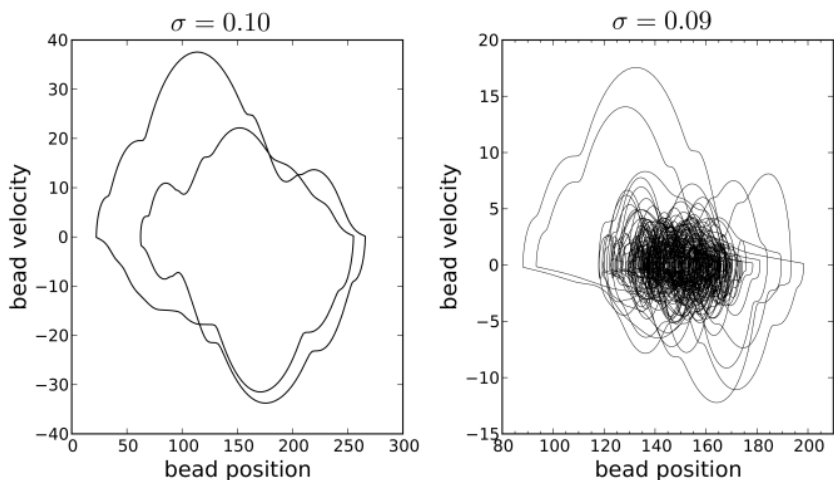

Figure 4. The phase portraits of the middle bead motion of the 5-bead chain for the "dissipation" parameter $\sigma$ taking the values 0.1 (left frame) and 0.09 (right frame). The period of the shown limit cycle is about 200; the chaotic phase portrait was obtained by visualizing the system motion within time interval about $5 \times 10^{5}$.

See Fig. 3. On one hand, this strong dependence explains that for chains of oscillators with not too weak "dissipation" only chaotic motion was found when the number of beads becomes sufficiently large, $N \gtrsim 100$ [5]. On the other hand, it enables us to pose a question about regarding the chaotic dynamics of such systems for $N \rightarrow \infty$ as a certain phase state.

In the case of weak "dissipation" the system dynamics exhibits sharp transition to a stable chaotic regime as the coefficient $\sigma$ decreases. It is demonstrated in Fig. 4 showing 
the transition from the regular dynamics for $\sigma=0: 1$ to a chaotic motion when $\sigma=0: 09$. As seen in Fig. 4 the chaotic portrait can be conceived of as a highly chaotic kernel surrounded by fragments of the regular limit cycle destroyed by instability.

Noise forces these systems to undergo two phase transitions as its intensity $\epsilon$ increases. The first one can be categorized as the transition from the regular bead motion to a cooperative chaotic bead motion. The latter means that the beads correlate substantially with one another in motion but individual trajectories are rather irregular and the magnitude of this irregularity cannot be due to the noise only. The second transition is determined by the formation of highly irregular mutually independent oscillations in the bead position. To illustrate the first phase transition Figure 5 depicts two phase portraits of the middle bead motion for different values of $\epsilon$. As seen, for $\epsilon=0: 01$ the phase portrait looks like a regular limit cycle disturbed by small noise. In contrast, when the noise intensity increases by two times, i.e., $\epsilon=0: 02$, the corresponding phase portrait becomes rather complex in form and the volume of the phase space layer containing the shown trajectory as a whole sharply grows.
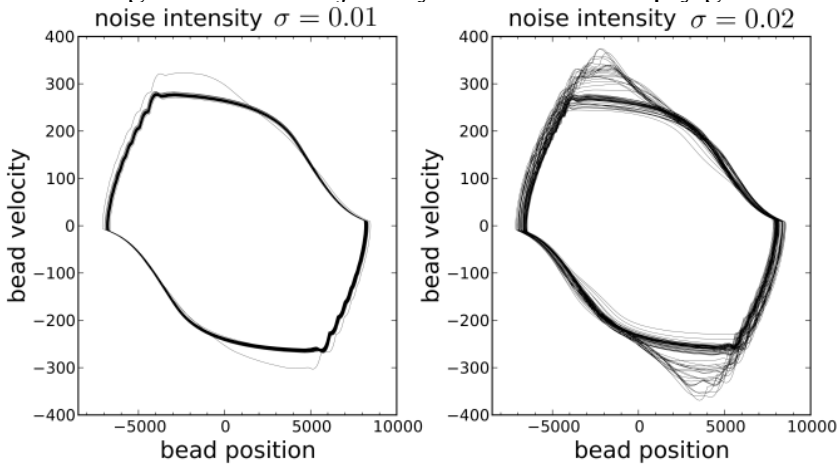

Figure 5. The phase portraits of the middle bead motion of the 30-bead chain with $\sigma=1$ for two values of the noise intensity $\epsilon=0.01$ and 0.02 . In plotting these portraits bead trajectories of motion during time interval about $2 \times 10^{4}$ were used.

\section{ACTION DYNAMICAL TRAP MODEL}

The developed dynamical traps model (3) reflects the effect of the equilibrium point indistinctness in humancontrolled dynamical processes. Still, one may argue that in such processes not only the desired end-state possesses the property of fuzziness, but also the desired action strategy is implemented in imperfect, non-optimal way. The purpose of the present section is to suggest a new mechanism based on the concepts of the original dynamical traps model that could potentially describe the effects of human inability or unwillingness to control dynamical systems precisely.

Keeping in mind model (3) let us define the operator control strategy as a linear feedback aimed at maintaining the system at the origin: $a(x, v)=x+\sigma v$ and the function $\Omega(v)$, Exp. (4), describes the operator fuzzy perception of the desired point $\{x=0, v=0\}$. In order to be able to capture the dynamical trap effect in controlling the deviations of current control parameter value from the optimal one, we extend the phase space of the system by regarding the acceleration $a=$ $d v / d t$ as an independent phase variable.
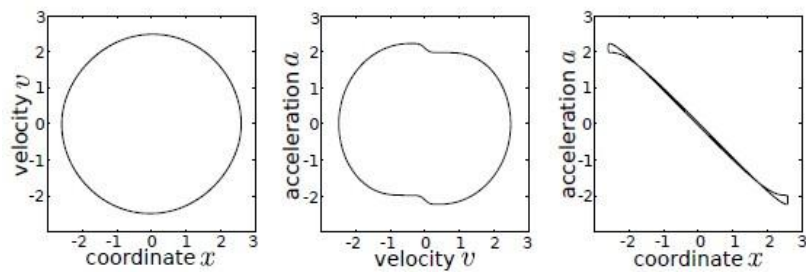

Figure 6. The projections of the limit cycle formed by the phase trajectory of system (6). The values of parameters used for simulation are $\sigma=1, \tau=$ 0:9.
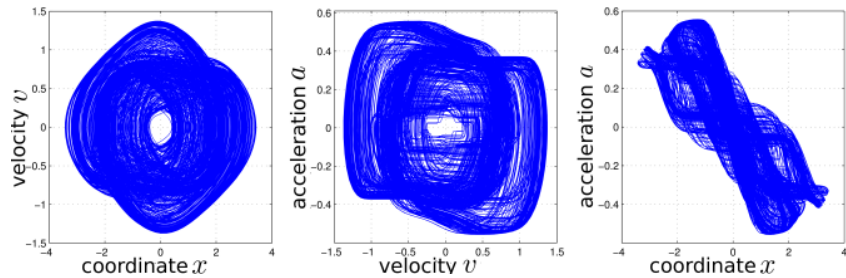

Figure 7. Phase space projections of the first particle motion in case of two oscillating particles. The following parameters were used for simulation: $\sigma_{0}=0: 01 ; \sigma=2 ; \tau=1 ; a_{t h}=1$

The proposed model is described by following equation

$$
\tau \frac{d a}{d t}=\Omega_{a}\left[a, a_{\mathrm{opt}}(x, v)\right]\left[a-a_{\mathrm{opt}}(x, v)\right]
$$

One may notice that if we set $\Omega_{a}\left[a, a_{\text {opt }}(x, v)\right] \equiv 1$, Eq. (6) describes following the optimal action strategy $a_{\text {opt }}:=a_{\text {opt }}(x$, $v$ ) precisely by the operator whose reaction time is $\tau$. However, as the human operator is not capable of doing so if the deviation is small, we set

$$
\Omega_{a}\left[a, a_{\mathrm{opt}}\right]=\frac{\Delta_{a} a_{\mathrm{th}}^{2}+\left(a-a_{\mathrm{opt}}\right)^{2}}{a_{\mathrm{th}}^{2}+\left(a-a_{\mathrm{opt}}\right)^{2}}
$$

where, in analogy to Exp. (4), $\Delta_{a}$ is the dynamical trap intensity coefficient and $a_{\mathrm{th}}$ is the threshold value of acceleration deviation from the optimal value. Finally, we specify the optimal control strategy as follows

$$
a_{\mathrm{opt}}(x, v)=\Omega(v)(x+\sigma v)
$$

Where the function $\Omega(v)$ is given by Exp. (4).

Two major patterns of system dynamics were observed depending on values of parameters. Namely, either the system exhibits periodic oscillations or the stable equilibrium point origin is the only attractor in the system phase space. The example of the found oscillation limit cycles is represented on Fig. 6. It is necessary to underline that the fuzzy perception of the optimal strategy causes the system instability on its own without noise. When the operator exhibits the fuzzy perception of the desired position only the 
instability arises due to the action of noise or the mismatch between the actions of other operators.

As done in the previous Section model (6) can be generalized to the chain of oscillators with action dynamical traps. Here we present the results of the numerical simulation of the system dynamics for the case of two oscillating particles. As can be seen in Fig. 7, the considered effect of humans imperfect perception of their own actions may cause irregular system motion patterns near the origin even in the systems with low number of particles; such phenomena were not observed previously. Various extremely complex patterns emerge in the system dynamics depending on the parameters. Moreover, the preliminary analysis of the system behavior under the presence of an additive noise revealed that the one-particle system dynamics patterns are stable with respect to the random perturbations, while the influence of the random factors on the behavior of larger particle ensembles is of more complex nature and requires an individual consideration.

\section{CONCLUSION}

The obtained results enable us to state that the bounded capacity of human cognition in ordering, e.g., events or actions can be regarded as a new mechanism of complex emergent phenomena in systems where human factor plays a crucial role. The mathematical formalism required for the description of this effect draws on the concept of dynamical traps being a certain alternative to the notion of stationary points in dynamical systems.

As far as the human fuzzy perception of the desired endstate is concerned, the model of oscillator with dynamical traps is proposed based on rather general assumptions about the actions of a human operator. It is demonstrated that in the chain of such oscillators complex emergent phenomena arise. They can be categorized as non-equilibrium phase transitions of a new type. In contrast to the phase transitions observed in physical systems they are due to the system stagnation in a certain region of the phase space rather than the regular "forces" changing their form.

A new model of human fuzzy rationality is proposed. A human controlling a dynamical system is usually not capable of selecting or calculating the optimal action strategy that would allow him to reach and maintain the desired end-state or goal. However, during the control process the operator is generally able to realize that the currently implemented strategy deviates from the optimal one if this deviation becomes large enough. Once being aware of the mismatch, the operator can adjust his actions until he feels that the current value of control parameter is acceptable. In order to capture this feature of human cognition, we, first, extend the phase space of the dynamical system under human control by regarding the particle acceleration, i.e. the control parameter as an independent phase variable. Second, we introduce a certain region alongside the optimal strategy in the space of all action strategies. The latter region is called the action dynamical trap. The existence of phase transitions induced by action dynamical traps is demonstrated.

\section{ACKNOWLEDGMENT}

The work was supported in part by the JSPS Grant 245404100001 .

\section{REFERENCES}

[1] Encyclopedia of Complexity and Systems Science, R. A. Meyers, Ed. SpringerScience+Buisiness Media, LLC., New York, 2009.

[2] R. R. Vallacher, "Social psychology, applications of complexity to," ibid. pp. 8420-8435.

[3] I. Lubashevsky, M. Hajimahmoodzadeh, A. Katsnelson, and P. Wagner, "Noise-induced phase transition in an oscillatory system with dynamical traps," Eur. Phys. J. B, vol. 36, pp. 11-118, 2003.

[4] I. Lubashevsky, R. Mahnke, M. Hajimahmoodzadeh, and A. A. Katsnelson, "Long-lived states of oscillator chains with dynamical traps," Eur. Phys. J. B, vol. 44, pp. 63-70, 2005.

[5] I. Lubashevsky, "Dynamical traps caused by fuzzy rationality," Adv. Com. Sys., vol. 15, p. 1250045(1-25), 2012.

[6] I. Lubashevsky, R. Mahnke, P. Wagner, S. Kalenkov, "Long-lived states in synchronized traffic flow: Empirical prompt and dynamical trap model," Phys. Rev. E,vol. 66, p. 016117(1-13) 2002.

[7] I. Lubashevsky, P. Wagner, R. Mahnke, "Bounded rational driver models," Eur. Phys. J. B, vol. 32, pp. 243-247,2003.

[8] I. Loram, H. Gollee, M. Lakie, and P. Gawthrop, "Human control of an inverted pendulum: is continuous control necessary? Is intermittent control effective? Is intermittent control physiological?", J. Physiol., vol. 589, pp. 307-324, 2011.

[9] J. L. Cabrera and J. G. Milton, "On-off intermittency in a human balancing task," Phys. Rev. Lett., vol. 89, p. 158702(1-4), 2002.

[10] M. Gestwa, "Using fuzzy control for modeling the control behavior of a human pilot," in: Fuzzy Controllers, Theory and Applications, L. Grigorie, Ed. InTech, 2011, Chap. 15, pp. 297-326.

[11] J. Milton, T. Ohira, J. Cabrera, R. Fraiser, J. Gyorffy, F. Ruiz, M. Strauss, E. Balch, P. Marin, and J. Alexander, "Balancing with vibration: A prelude for "Drift and Act" balance control," PLoS One, vol. 4, p. e7427(1-11), 2009.

[12] I. Loram, C. Maganaris, and M. Lakie, "Human postural sway results from frequent, ballistic bias impulses by soleus and gastrocnemius,", J. Physiol., vol. 564, pp. 295-311, 2005.

[13] A. Zgonnikov, I. Lubashevsky, and M. Mozgovoy, "Computer simulation of stick balancing: action point analysis." Proceed. 2012 Joint International Conference on

[14] Human-Centered Computer Environments, V. Klyuev, A. Vazhenin, J. Herder, K. Miura, Eds. ACM, New York, NY, USA, 2012, pp. 162164. 